\title{
Trichophyton verrucosum
}

National Cancer Institute

\section{Source}

National Cancer Institute. Trichophyton verrucosum. NCI Thesaurus. Code C127758.

A species of zoophilic and dermatophytic fungi in the phylum Ascomycota. This species causes ringworm in cattle. 